\title{
Evaluation of Hydrodynamic Pressure Distribution in Reservoir of Concrete Gravity Dam under Vertical Vibration Using an Analytical Solution
}

\author{
Majid Pasbani Khiavi $\mathbb{D}^{1}$ and Ali Sari $\mathbb{D}^{2}$ \\ ${ }^{1}$ Faculty of Engineering, University of Mohaghegh Ardabili, Ardabil, Iran \\ ${ }^{2}$ Faculty of Civil Engineering, Istanbul Technical University, Istanbul 34467, Turkey \\ Correspondence should be addressed to Majid Pasbani Khiavi; pasbani@uma.ac.ir
}

Received 12 November 2020; Revised 18 April 2021; Accepted 29 April 2021; Published 7 May 2021

Academic Editor: Marjan Uddin

Copyright (c) 2021 Majid Pasbani Khiavi and Ali Sari. This is an open access article distributed under the Creative Commons Attribution License, which permits unrestricted use, distribution, and reproduction in any medium, provided the original work is properly cited.

\begin{abstract}
Fluid-structure interaction causes a hydrodynamic force, which can be exerted to the dam and affects its response. The effect of vertical excitation of ground motion on dynamic behavior of concrete gravity dam is the most important because of the interaction between foundation and reservoir. So, the foundation-reservoir interaction should be taken into account in designing concrete dams. In most studies, the effects of the vertical component of vibration have been ignored. While in vertical vibration, due to the interaction of the reservoir and the foundation, a significant hydrodynamic pressure is produced in the tank, which increases the dam response. In this study, the hydrodynamic pressure wave propagation in the reservoir of a concrete gravity dam caused by interaction with the foundation under vertical vibration is investigated using an analytical method. To achieve an analytical solution, the reservoir is assumed to be rectangular, and a harmonic load is vertically applied on the system from the foundation. Considering the acoustic nature of the reservoir fluid under harmonic vibration, a new method using the separation of variables method has been used for solution of hydrodynamic wave equation. The results show a significant effect of the vertical component of earthquake on the amount of induced pressure distributed in the reservoir, which has been omitted in most previous studies. Obtained results of the proposed model can be extended to more complicated models in terms of different loading and geometrical conditions.
\end{abstract}

\section{Introduction}

Fluid-structure interaction is one of the most extensive discussions concerning the behavior of fluid, and numerous research works are performed on this topic. Fluid-structure interaction occurs when there is a relative motion between the two systems. The dynamic response of systems under the effect of the fluid-structure interaction is of great importance.

During the ground motion, the interaction of the reservoir with dam and foundation can produce a hydrodynamic pressure in the reservoir. Hence, besides the inertia force due to the ground movement, the hydrodynamic force on the upstream face of the dam is one of the main factors exerting a load on the dam structure in the event of a vibration. Neglecting the effects of hydrodynamic force will result inaccurate responses, which can bring some challenges for the designed structure. In other words, taking into account the fluid-structure interaction in the dynamic analysis of concrete dams will increase the accuracy of the responses obtained from the analysis and better estimation of hydrodynamic forces and the stresses created in the dam body.

The first model concerning the investigation of the interaction effect in the seismic behavior of dams was proposed by Westergaard in 1933 [1]. In this model, the damreservoir interaction under the effect of horizontal vibrations was proposed considering the two-dimensional model. In the Westergaard model, the dam has been assumed to be rigid with a semilimited reservoir and a constant depth, and 
the fluid pressure distribution in the dam-reservoir interface was extracted by the analytical method. Westergaard showed that the induced force because of interaction is proportional to the acceleration caused by the horizontal excitation and can be estimated by a parabolic distribution on the dam height. His method is called the added mass approximation. An important point in Westergaard solution is that the maximum energy caused by the seismic motion is related to the frequencies that are smaller than the natural frequency of the reservoir. Several research studies show that the hydrodynamic pressure distribution caused by an earthquake on the dam body is almost parabolic.

In the Westergaard added mass model, the effect of compressibility of water has been neglected. However, the accurate fluid structure analyses have shown that the compressibility of water has a considerable effect on the hydrodynamic pressure and dynamic behavior of concrete dam. In the dam-reservoir system, the effects of water compressibility are very important when the fundamental frequency of dam is close to the natural excitation frequency of the reservoir. The reason for less attention to the compressibility effects of water is that the investigation of fluidstructure interaction is only necessary for problems related to the wave propagation, and the propagation of the hydrodynamic waves only happens in the compressible flow. If the compressibility effects are neglected, an incompressible model can be used in which the propagation of hydrodynamic waves is neglected.

Chopra investigated the effects of fluid compressibility on the fluid structure interaction and induced force [2]. His model was similar to the Westergaard model. Chopra concluded that in a case of neglecting of water compressibility, a significant error is appeared in the analysis results. In most of the studies, the incompressibility assumption has been used for water because in this case, the modeling of the dam-reservoir system will be simplified. Therefore, in this condition, there is no need to solve complicated integrals.

In the following, Chopra studied the effect of dam flexibility on the interaction forces by modeling the dam as a system which composes of mass, damper, and spring [3]. He extracted the parameters of the dam model by focusing on the first special mode of a triangle-shaped dam. He showed that natural frequencies of the dam-reservoir coupled system are different with than that of the uncoupled system. Chopra found that if the ratio of fundamental frequency of dam to natural frequency of the reservoir is higher than two, the compressibility effect of the water can be neglected. In the Chopra model, dam was only under the effect of horizontal acceleration of the ground motion. In 1970, Chopra analyzed the dam-reservoir model to calculate the dam response considering the interaction effect [4]. He showed that by considering the effects of the dam-reservoir interaction, the response of dam significantly increases in comparison with a case in which the effect of interaction is neglected.

There are few works that addressed the investigation of dynamic pressure distribution in a reservoir under the effect of the vertical component. As an example, in 1971, Nath proposed a model in which the dam was considered as a cantilever beam which has been exposed under the shear and bending effects [5]. By applying the vertical component of the earthquake, the effect of vertical vibration of ground motion on the dam-reservoir interaction was investigated as a $1 \mathrm{D}$ model using the finite element method. Nath showed that although the hydrodynamic pressure produced on the dam due to the vertical vibration in a specific frequency is considerably higher than the pressure caused by the horizontal movement under the same frequency, but the displacement response of the structure is higher for the horizontal movement.

Chakrabarti and Chopra approximated the response of the dam under vertical earthquake component by the deformations in the fundamental mode of vibration considering the flexibility of bed rock using the analytical model. Obtained results illustrated that the hydrodynamic forces induced by vertical vibration are affected by dam-reservoir interaction and depend on the flexibility of the dam. They showed that the vertical component of ground motion induces significant hydrodynamic forces in the horizontal direction on a vertical upstream face. So, it is very important to consider the effect of the vertical component in seismic analysis of concrete gravity dams [6].

In 1988, Humar and Jablonski presented a model in the frequency domain to investigate the hydrodynamic pressure distribution in a reservoir under the effect of vertical component of the earthquake using the boundary element method [7]. They show that the amount of hydrodynamic pressure produced in the reservoir under the effect of the vertical component of the earthquake is more than that of the horizontal component.

In recent years, some research studies have been performed on the dynamic behavior of the concrete gravity dam using the analytical models. Ghaemian et al. investigated the nonlinear seismic behavior of a concrete gravity dam by considering the heterogeneity and displacement of the foundation [8]. The obtained results from the model show that the heterogeneity of the foundation can affect the system response and causes the rapid occurrence of a crack in the dam body during the earthquake. Ziaolhagh et al. analyzed the dam and foundation system by using the finite element method with triangle elements and 21-point Gaussian integration of the dam system [9]. They assumed that the dam is flexible, and the system frequencies and mode shape were extracted using the finite element method. They compared the results of 8-node and 21-node elements and showed that the accuracy of the finite element model with 21-node element is higher. Ribeiro and Pedroso provided a review on the analysis method for concrete gravity dams under the effect of seismic load [10]. They studied the limits of the previous methods by the finite element method and extracted the dam response using the semistatic, semidynamic and dynamic methods.

Some research has examined the effects of heat transfer on solid contact. Some recent studies have used nanofluids and nanoparticles to more accurately study the partial specifications of water and the state of fluid temperature changes which can be referred to studies by Zahir Shah et al. in 2020, Dawar et al., Khan et al., and Nasir et al. research [11-16]. 
However, according to the conditions of the dam-reservoir, the thermal changes of the water are not important, the water density is assumed to be constant, and the foundation is impenetrable.

Studying of the previous research studies illustrate that the investigation of the hydrodynamic pressure distribution caused by the foundation-reservoir interaction due to the vertical vibration of the ground motion in the concrete gravity dams has been less evaluated. In other words, the importance of attention to vertical vibration using the numerical and analytical methods is among the topic which has received less attention in previous research.

So, in this study, the hydrodynamic pressure propagation in the reservoir of a concrete gravity dam caused by reservoir and foundation interaction under the vertical excitation is investigated using an analytical model. In the analytical method, some loading and geometrical simplifications may be performed on the model, but because of the response accuracy and the ability to perform the parametric study of variables, it can be a capable practice in studying the interaction effects and hydrodynamic behavior of reservoir. Also, it should be noted that the numerical methods require the coding and high computational efforts, and they are used when obtaining the analytical solution for the problem is impossible.

\section{Dynamic Equation of the Fluid inside the Reservoir}

In the problems concerning the fluid-structure acoustic interaction, the dynamic equation of structure should be along with the Navier-Stokes and continuity equations of the fluid. To obtain the governing equation, the fluid inside the reservoir, the following assumptions are credible in the dam-reservoir system $[3,6]$ :

(i) The fluid is acoustic and can be assumed to be nonviscous with constant entropy, so that the fluid particles undergo small deformations

(ii) Given the specifications of the reservoir, the water density variation is negligible

(iii) The velocity values are so small that its transitional effects can be neglected

(iv) The water viscosity is small, so the shear stresses can be neglected

By applying the assumptions, the continuity equations can be simplified as follows:

$$
\rho \nabla^{T} V+\frac{\partial \rho}{\partial t}=0 .
$$

The relationship between the fluid density and the produced hydrodynamic pressure is defined by the equation of state:

$$
\mathrm{d} \rho=\frac{\rho}{k} \mathrm{~d} P
$$

where $k, \rho, P, V$, and $t$ denote the coefficient of fluid volume changes, fluid density, fluid pressure, fluid velocity vector, and time. From equations (1) and (2), it can be written as

$$
\nabla^{T} V=-\frac{1}{k} \frac{\partial P}{\partial t} .
$$

Neglecting the body gravity effects and based on the assumptions made, the momentum equation of the fluid is summarized as follows:

$$
\rho \dot{V}+\nabla P=0
$$

The gradient of momentum equation can be written as

$$
\rho \nabla^{T} \dot{V}+\nabla^{2} P=0 .
$$

According to equations (3) and (5), the following equation is obtained:

$$
\nabla^{2} P=\frac{\rho}{k} \frac{\partial^{2} P}{\partial^{2} t}
$$

or

$$
\nabla^{2} P=\frac{1}{C^{2}} \frac{\partial^{2} P}{\partial^{2} t},
$$

where $C=\sqrt{k / \rho}$ denotes the speed of acoustic waves within the fluid. Equation (7) is the governing equation of the acoustic fluid inside the reservoir which is known as Helmholtz equation.

If the fluid is assumed to be incompressible, the Helmholtz equation becomes the Laplace equation:

$$
\nabla^{2} P=0
$$

For solving the Helmholtz equation, the appropriate boundary conditions are required which will be addressed in the following [1-4].

\section{Boundary Condition}

3.1. Reservoir Bed Boundary. To take into account the interaction effects, the boundary condition of the foundationreservoir interface must be extracted. If no absorption or penetration of water is occurred by the foundation, the boundary condition at the bottom of the reservoir is similar to the boundary condition at the fluid-solid interface. But, in the case of existence of water absorption at the bottom, its corresponding boundary condition can be extracted [17]. Given that the shear strength of water is negligible, in such case, the interaction in the vertical direction can only be considered where the hydrodynamic pressure waves influences the bottom of reservoir only in the vertical direction. According to the discussions, it is clear that the hydrodynamic pressure in the water $P(n, t)$ in a one-dimensional case can be defined using the wave equation:

$$
\frac{\partial^{2} P}{\partial n^{2}}=\frac{1}{C^{2}} \frac{\partial^{2} P}{\partial t^{2}}, \quad n \geq 0 .
$$


Similarly, displacement due to the interaction of reservoir and foundation, $x(n, t)$, in the reservoir bottom can be defined as follows:

$$
\frac{\partial^{2} x}{\partial n^{2}}=\frac{1}{C_{f}^{2}} \frac{\partial^{2} x}{\partial t^{2}}, \quad n \leq 0
$$

where $C_{f}=\sqrt{E_{f} / \rho_{f}}$.

$E_{f}$ is the modulus of elasticity, and $\rho_{f}$ is the density of the materials of the reservoir bottom. At the bottom of the reservoir, according to the Euler equation, the vertical pressure gradient is proportional to the total acceleration of the foundation. Hence,

$$
\frac{\partial P(0, t)}{\partial n}=-\rho\left[a_{n}(t)+\ddot{x}(0, t)\right] .
$$

$\ddot{x}(0, t)$ is the acceleration of reservoir bed induced by reservoir-foundation interaction. The equilibrium of particles in the reservoir bed causes the following equation:

$$
P(0, t)=-E_{f} \frac{\partial x}{\partial n}(0, t)
$$

The d'Alembert solution for equation (10) is $x=$ $g_{f}\left(n+C_{f} t\right)$, where $g_{f}$ is the defining function of impacted wave amplitude that moves vertically downward in the materials of the reservoir bottom. By using this relation, it can be written as

$$
\frac{\partial x}{\partial t}(0, t)=C_{f} g_{f}^{\prime}\left(C_{f} t\right)
$$

and hence,

$$
\ddot{x}(0, t)=C_{f}^{2} g_{f}^{\prime \prime}\left(C_{f} t\right),
$$

where the prime sign denotes the derivative of $g_{f}$ respect to $\left(n+C_{f} t\right)$ parameter. By differentiating equation (12) and using equation (14), the following equation is obtained:

$$
\frac{\partial P(0, t)}{\partial t}=-E_{f} C_{f} g_{f}^{\prime \prime}\left(C_{f} t\right)
$$

or

$$
\frac{\partial P(0, t)}{\partial t}=-\frac{E_{f}}{C_{f}} \ddot{x}(0, t) .
$$

From equations (11) and (16),

$$
\frac{\partial P(0, t)}{\partial n}=-\rho\left[a_{n}(t)-\frac{C_{f}}{E_{f}} \frac{\partial P(0, t)}{\partial t}\right],
$$

where $q=\left(\rho_{w} / \rho_{f} C_{f}\right)$ is the attenuation coefficient.

If the reservoir bed is assumed horizontal, the corresponding boundary condition is defined as follows:

$$
\frac{\partial P}{\partial y}+q \dot{P}=-\rho a_{y}(x, t) \text {, on } L_{r f},
$$

where $L_{r f}$ is the boundary of the reservoir with foundation interface.

For rigid reservoir bottom $C_{f}=\infty$ and $q=0$. Hence, the second term of equation (18) is eliminated, and the boundary condition for the rigid bottom of the reservoir is obtained:

$$
\frac{\partial P}{\partial y}=-\rho a_{y}(x, t) \text {, on } L_{r f} .
$$

3.2. Reservoir Free Surface Boundary. If the effect of the gravitational waves is neglected, the boundary condition of the free surface is defined as follows:

$$
P=0 \text {, on } L_{f},
$$

where $L_{f}$ is the boundary corresponding to the free surface.

3.3. Dam-Reservoir Interface Boundary. To apply the interaction effects at the fluid-solid interface, it is necessary that the dam acceleration be related to reservoir pressure produced in the reservoir. For this purpose, by using the force balance relation in the contact surface, the boundary condition is written as follows

$$
\frac{\partial P}{\partial x}=-\rho_{w} a_{x} \text {, on } L_{r d}
$$

where $\rho_{w}, L_{r d}, a_{x}$, and $(\partial P / \partial x)$ are the water density, damreservoir contact boundary, outward acceleration normal to the boundary, and the hydrodynamic pressure gradient along the vector normal to the interface, respectively. Given that, this study aims to analyze the reservoir under the vertical vibration; hence, it is assumed that $a_{x}=0$, and the interface boundary condition is defined as follows:

$$
\frac{\partial P}{\partial x}=0, \text { on } L_{r d}
$$

3.4. Far-End Boundary Condition. Given the upward propagation of the hydrodynamic wave far from the reservoir, it is necessary that the pressure is completely dissipated, and there is no reflection from the far boundary. So,

$$
P=0 \text {, on } L_{\text {Infinite }} \text {. }
$$

where $L_{\text {Infinite }}$ corresponds to the far end of reservoir, in which $x \longrightarrow \infty$ in analytical solution.

\section{Analytical Solution}

By using the extracted equation for the system under the fluid-structure interaction and the boundary conditions corresponding to the domains, the response of the desired system can be obtained by the analytical solution.

To achieve an analytical solution process, the reservoir system is considered as $2 \mathrm{D}$ with rectangular shaped. The wave equation extracted in the previous sections can be used for the reservoir. Therefore, the behavior of the water inside the reservoir can be defined by the wave equation in terms of pressure variable: 


$$
\frac{\partial^{2} P}{\partial x^{2}}+\frac{\partial^{2} P}{\partial y^{2}}=\frac{1}{C^{2}} \frac{\partial^{2} P}{\partial t^{2}}, \text { in } S
$$

where $C$ is the acoustic wave speed, $S$ is the reservoir domain, and $P$ is the hydrodynamic pressure created in the reservoir.

Since wave equation is a second-order differential equation, the appropriate boundary conditions are required for solving.

One way to solve the wave equation in applied mathematics is to use the separation of the variables method [18].

For this purpose, it is assumed that the pressure response is defined as the product of three independent functions, one dependent on the parameter $x$, and the others dependent on the parameters $y$ and $t$. That means,

$$
P=P_{x} P_{y} P_{t} \text {. }
$$

Due to the independence of $x, y$, and $t$, second derivative of $P_{x}, P_{y}$ and $P_{t}$ can be expressed as follows:

$$
\begin{aligned}
& \frac{\partial^{2} P}{\partial x^{2}}=P_{x}^{\prime \prime} P_{y} P_{t}, \\
& \frac{\partial^{2} P}{\partial y^{2}}=P_{x} P_{y}^{\prime \prime} P_{t}, \\
& \frac{\partial^{2} P}{\partial t^{2}}=P_{x} P_{t} P_{t}^{\prime \prime} .
\end{aligned}
$$

The following result is obtained by placing the equations in the governing wave equation of reservoir:

$$
P_{x}^{\prime \prime} P_{y} P+P_{x} P_{y}^{\prime \prime} P_{t}=\frac{1}{C^{2}} P_{x} P_{y} P_{t}^{\prime \prime} .
$$

So,

$$
\frac{P_{x}^{\prime \prime}}{P_{x}}+\frac{P_{y}^{\prime \prime}}{P_{y}}=\frac{1}{C^{2}} \frac{P_{t}^{\prime \prime}}{P_{t}}
$$

According to equation (28) and the independence of functions, to make a relationship, $\left(P_{x}^{\prime \prime} / P_{x}\right),\left(P_{y}^{\prime \prime} / P_{y}\right)$, and $\left(P_{t}^{\prime \prime} / P_{t}\right)$ must have a constant value. Therefore, because of the dynamic nature of the problem, the following conditions can be achieved:

$$
\begin{aligned}
& \frac{P_{x}^{\prime \prime}}{P_{x}}=-k^{2}, \\
& \frac{P_{y}^{\prime \prime}}{P_{y}}=-\lambda^{2}, \\
& \frac{P_{t}^{\prime \prime}}{P_{t}}=-\omega^{2} .
\end{aligned}
$$

Therefore,

$$
-k^{2}-\lambda^{2}=-\frac{1}{C^{2}} \omega^{2}
$$

or

$$
\lambda^{2}=\frac{\omega^{2}}{C^{2}}-k^{2} .
$$

According to the mentioned equation and considering the dynamic nature of the pressure wave and due to the propagation of the wave to the far and its dissipation, the solutions to the problem can be considered as follows:

$$
\frac{P_{x}^{\prime \prime}}{P_{x}}=-k^{2} \Rightarrow P_{x}=A_{1} e^{-i k x}+A_{2} e^{i k x} .
$$

By applying the far end boundary condition of the reservoir $(x \longrightarrow \infty \Rightarrow P=0), A_{2}$ must be equal to zero, and the following result is obtained:

$$
\begin{gathered}
P_{x}=A_{1} e^{-i k x}, \\
P(x, y, t)=P_{y} P_{t} A_{1} e^{-i k x} .
\end{gathered}
$$

By using the dam-reservoir interface condition $(x=0 \Rightarrow(\partial P / \partial x)=0)$, it can be written as

$$
\begin{aligned}
\frac{\partial P}{\partial x}= & -k P_{y} P_{t} A_{1} e^{-i k x} \\
& -k P_{y} P_{t} A_{1}=0
\end{aligned}
$$

For the satisfying the above condition, it is necessary that $k=0$.

So, it can be concluded that in as case, a vertical vibration is applied to the reservoir bottom; as expected, the produced hydrodynamic pressure is independent of horizontal direction. By merging the constant $A_{1}$ into other coefficients, the hydrodynamic pressure response is calculated as

$$
P=P_{y} P_{t}
$$

$$
\begin{aligned}
& \frac{P_{t}^{\prime \prime}}{P_{t}}=-\omega^{2} \Rightarrow P_{t}=B_{1} e^{-i \omega t}+B_{2} e^{i \omega t}, \\
& \frac{P_{y}^{\prime \prime}}{P_{y}}=-\lambda^{2} \Rightarrow \frac{\partial^{2} P_{y}}{\partial y^{2}}+\lambda^{2} P_{y}=0,
\end{aligned}
$$

where $\lambda^{2}=\left(\omega^{2} / C^{2}\right)-k^{2}$.

The general solution of equation (37) is as follows:

$$
P_{y}=C_{1} \operatorname{Cos} \lambda y+C_{2} \operatorname{Sin} \lambda y,
$$

where $C_{1}$ and $C_{2}$ are the constants obtained by the boundary conditions. By substituting the free surface boundary condition ( $y=H \Rightarrow P=0$ ) in equation (38),

$$
P_{y}(H)=C_{1} \operatorname{Cos} \lambda H+C_{2} \operatorname{Sin} \lambda H=0 .
$$

Hence, $C_{2}$ is obtained as

$$
C_{2}=-C_{1} \operatorname{Cot} \lambda H \text {. }
$$

So, by applying the free surface boundary condition, the general solution is defined as

$$
P_{y}(y)=C_{1}(\operatorname{Cos} \lambda y-\operatorname{Cot} \lambda H \operatorname{Sin} \lambda y),
$$


or

$$
\begin{aligned}
& P_{y}(y)=C_{1} \frac{\operatorname{Sin} \lambda(H-y)}{\operatorname{Sin} \lambda H}, \\
& P_{y}^{\prime \prime}(y)=-C_{1} \lambda^{2} \frac{\operatorname{Sin} \lambda(H-y)}{\operatorname{Sin} \lambda H} .
\end{aligned}
$$

By applying the boundary condition of the foundationreservoir interface, equation (18) to equation (42), and by assuming that the harmonic vertical acceleration is $a_{y}=\widehat{a}_{y} e^{i \omega t}$, it can be found that

$$
-C_{1} \lambda \frac{\operatorname{Cos} \lambda(H-y)}{\operatorname{Sin} \lambda H} P_{t}=-\rho \widehat{a}_{y} e^{i \omega t}-C_{1} i \omega q \frac{\operatorname{Sin} \lambda(H-y)}{\operatorname{Sin} \lambda H} P_{t}, \quad y=0,
$$

or

$$
-C_{1} \lambda \operatorname{Cot} \lambda H P_{t}=-\rho \widehat{a}_{y} e^{i \omega t}-C_{1} i \omega q P_{t} .
$$
that

Considering that $P_{t}=B_{1} e^{-i \omega t}+B_{2} e^{i \omega t}$, it can be written

$$
\begin{aligned}
-C_{1} \lambda \operatorname{Cot} \lambda H\left(B_{1} e^{-i \omega t}+B_{2} e^{i \omega t}\right)= & -\rho \widehat{a}_{y} e^{i \omega t} \\
& -C_{1} i \omega q\left(B_{1} e^{-i \omega t}+B_{2} e^{i \omega t}\right) .
\end{aligned}
$$

For satisfying the above equation, $B_{1}$ constant must be equal to zero $\left(B_{1}=0\right)$ and so

$$
\begin{array}{r}
P_{t}=B_{2} e^{i \omega t}, \\
-C_{1} \lambda \operatorname{Cot} \lambda H=-\rho \widehat{a}_{y}-C_{1} i \omega q .
\end{array}
$$

In the above equation, $B_{1}$ constant has been merged with $C_{1}$, and $C_{1}$ is obtained as follows:

$$
C_{1}=\frac{\rho \widehat{a}_{y}}{\lambda \operatorname{Cot} \lambda H-i \omega q} .
$$

So, $P_{y}$ is obtained as

$$
P_{y}=\frac{\rho \widehat{a}_{y}}{\lambda \operatorname{Cot} \lambda H-i \omega q} \frac{\operatorname{Sin} \lambda(H-y)}{\operatorname{Sin} \lambda H} .
$$

The peak values of this function occurred at frequencies of $(\omega H / C)=(\pi / 2),(3 \pi / 2),(5 \pi / 2), \ldots$

So,

$$
P=\frac{\rho \widehat{a}_{y}}{\lambda \operatorname{Cot} \lambda H-i \omega q} \frac{\operatorname{Sin} \lambda(H-y)}{\operatorname{Sin} \lambda H} e^{i \omega t} .
$$

Obtained response is similar to result presented by Chakrabarti and Chopra (1973 and 1983) $[6,19]$.

The specific cases of the extracted function can be corresponding to the rigid foundation and incompressible fluid.

4.1. Incompressible Fluid. If the fluid is incompressible, then $\omega \longrightarrow 0$ and $\lambda \longrightarrow 0$. In this case, the limit of the $P$ function is defined as follows:

$$
P=\frac{\rho \widehat{a}_{y}}{\lambda} \frac{\operatorname{Sin} \lambda(H-y)}{\operatorname{Cos} \lambda H}=\frac{\rho \widehat{a}_{y}}{\operatorname{Cos} 0 \times H} \operatorname{Lim} \frac{\operatorname{Sin} \lambda \times(H-y)}{\lambda},
$$

when $\lambda \longrightarrow 0$. Then, $\operatorname{Lim}(\operatorname{Sin} \lambda \times(H-y) / \lambda)=(\lambda \times$ $(H-y) / \lambda)$ or $(H-y)$.

Therefore, the compressive response is summarized as follows:

$$
P \longrightarrow \rho \widehat{a}_{y}(H-y),
$$

which denotes the linear distribution for pressure.

Obtained solution for incompressible fluid compiles with the pressure distribution equation in the vertical direction defined in hydrodynamic is $\left((\partial P / \partial y)=-\rho \widehat{a}_{y}\right)$.

According to this relation, it can be found that in a case where the fluid is incompressible, the pressure distribution in the reservoir under the effect of the vertical acceleration is not depending on the bottom absorption and the time.

4.2. Rigid Foundation. In case where the foundation is rigid, $q=0$, and the hydrodynamic pressure is summarized as follows:

$$
P=\frac{\rho \widehat{a}_{y}}{\lambda \operatorname{Cot} \lambda H} \frac{\operatorname{Sin} \lambda(H-y)}{\operatorname{Sin} \lambda H} e^{i \omega t},
$$

or

$$
P=\frac{\rho \widehat{a}_{y}}{\lambda} \frac{\operatorname{Sin} \lambda(H-y)}{\operatorname{Cos} \lambda H} e^{i \omega t} .
$$

The maximum values for this function have occurred at frequencies of $(\omega H / C)=(\pi / 2),(3 \pi / 2),(5 \pi / 2), \ldots$.

\section{Concrete Gravity Dam and Reservoir Model}

The proposed analytical solution is investigated for a case study model, and the response for different parameters is examined. The geometric characteristic of the model, which is a concrete gravity dam with $100 \mathrm{~m}$ height of reservoir, is shown in Figure 1. A harmonic vertical acceleration of $a_{y}=$ $\widehat{a}_{y} e^{i \omega t}$ with $\widehat{a}_{y}=0.5 \mathrm{~g}$ has been applied to model.

The water density and speed of compressive waves in the water have been considered as $1000 \mathrm{Kg} / \mathrm{m}^{3}$ and $1438.66 \mathrm{~m} / \mathrm{s}$, respectively. To investigate the foundation effect, the modulus of elasticity and the density of foundation are assumed to be $3430 \mathrm{MPa}$ and $2400 \mathrm{Kg} / \mathrm{m}^{3}$, respectively [20].

\section{Results}

Using the extracted analytical solution, the responses for rigid and flexible foundation were obtained and are presented in Figures 2-5. For this purpose, the responses have been separated for the frequency less and more than the first natural frequency of reservoir $(\Omega=(\pi \mathrm{C} / 2 \mathrm{H}))$, and the hydrodynamic pressure distribution in the dam-reservoir interface against the height is shown. It should be mentioned that $y$ is the water depth of respect to the reservoir bottom in terms of meter. 


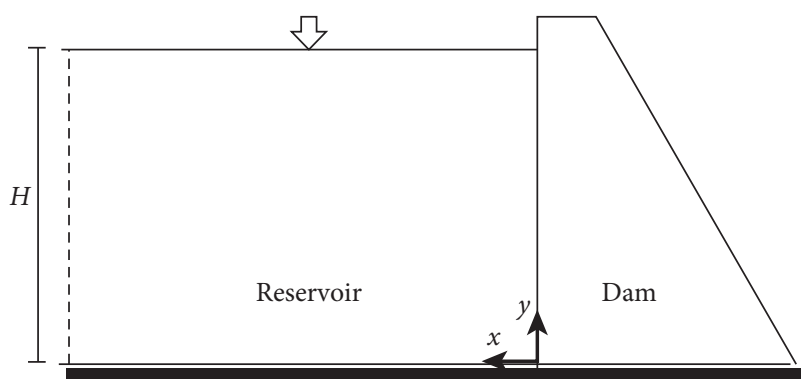

FIgURE 1: Concrete gravity dam geometry.

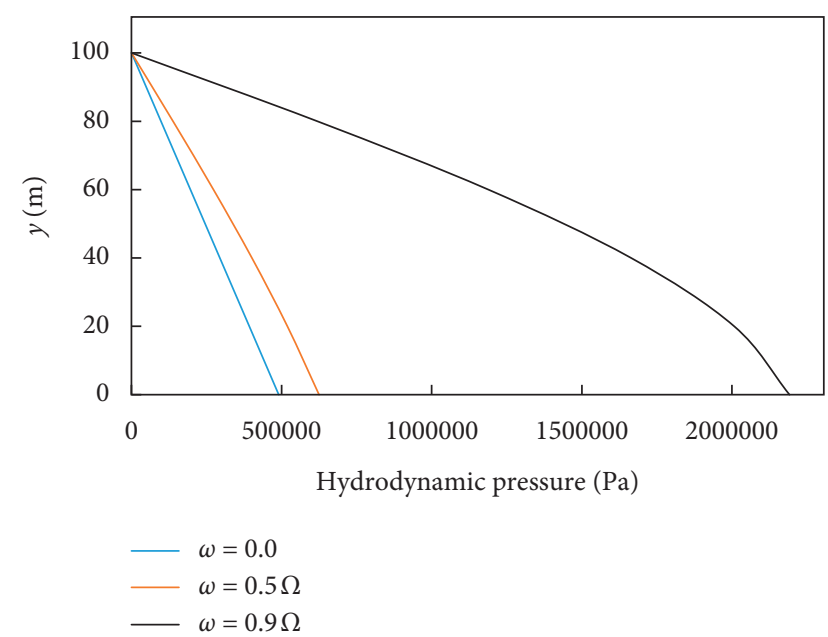

Figure 2: Pressure distribution along the dam height for frequencies less than the natural frequency of the reservoir in rigid foundation case.

According to the presented curves, the trend of hydrodynamic pressure distribution under vertical vibration can be discussed.

The first interesting case in Figure 2 is for the case in which $\omega=0$, that is related to the condition of incompressible fluid and indicates that the pressure distribution in this case is linear.

Also, by comparing Figure 2 with Figure 3 and Figure 4 with Figure 5, it can be seen that for a case in which the excitation frequency is more than the natural reservoir frequency $(\omega>\Omega)$, unlike the case in which the excitation frequency is less than the natural reservoir frequency $(\omega<\Omega)$, the maximum hydrodynamic pressure is not created at the bottom of the dam and occurred near the $y=0.3 H$.

Another interesting case is the case $\omega=2.0 \Omega$ in Figures 3 and 5 , where the pressure at the bottom of the reservoir is zero, which is due to the absorption of the excitation with the natural vibration of the reservoir.

The analysis of pressure distribution in the figures shows that the effect of compressibility for the case in which the excitation frequencies are less than half of the natural frequency of the reservoir $(\omega<0.5 \Omega)$ are not very important. This has been mentioned in previous research for horizontal excitation.

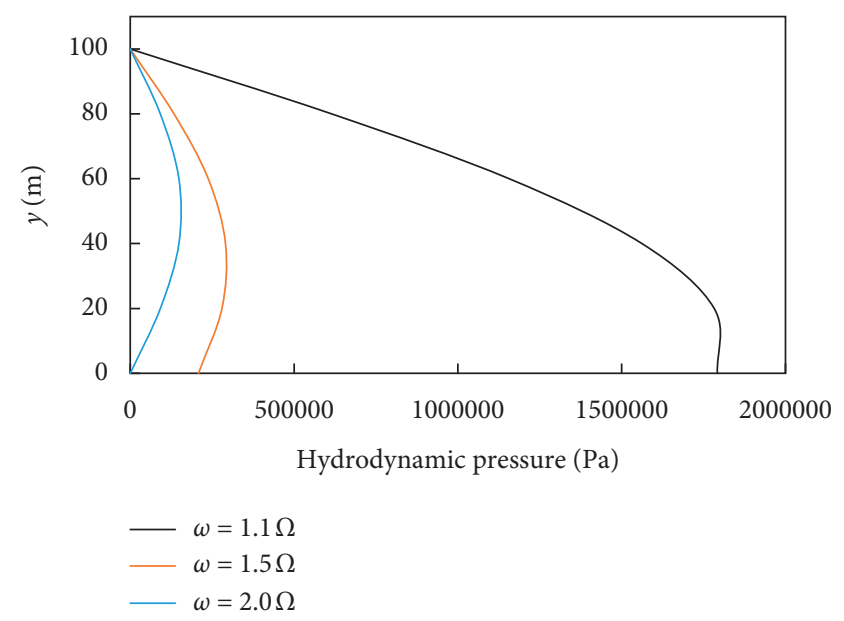

FIgURE 3: Pressure distribution along the dam height for frequencies more than the natural frequency of the reservoir in rigid foundation case.

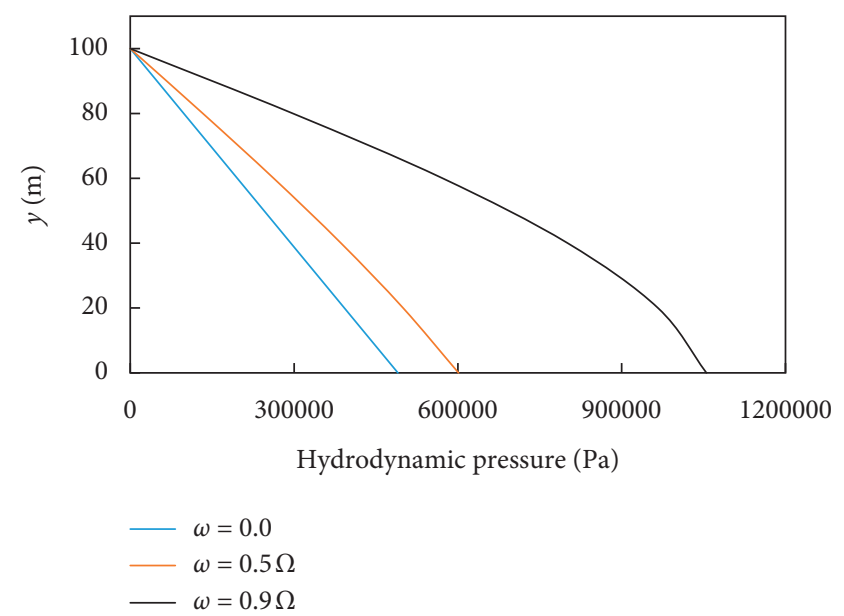

FIgURE 4: Pressure distribution along the dam height for frequencies less than the natural frequency of the reservoir in flexible foundation case.

In order to investigate the effect of excitation frequency on the hydrodynamic pressure distribution process, the hydrodynamic pressure sensitivity curve concerning the excitation frequency was extracted for both rigid and flexible foundation and isshown in Figures 6 and 7.

According to the curves, it can be found that the maximum responses of the system are corresponding to a case in which the excitation frequency is close to the natural frequencies of the reservoir $(\omega=(n \pi C / 2 H))$.

\section{Discussion}

Also in some studies, to simplify the analysis, the foundation is assumed to be rigid; that assumption is not correct because the foundation under the dam and reservoir was flexible and its movement produces hydrodynamic pressure in the reservoir due to the interaction of the dam and the reservoir. 


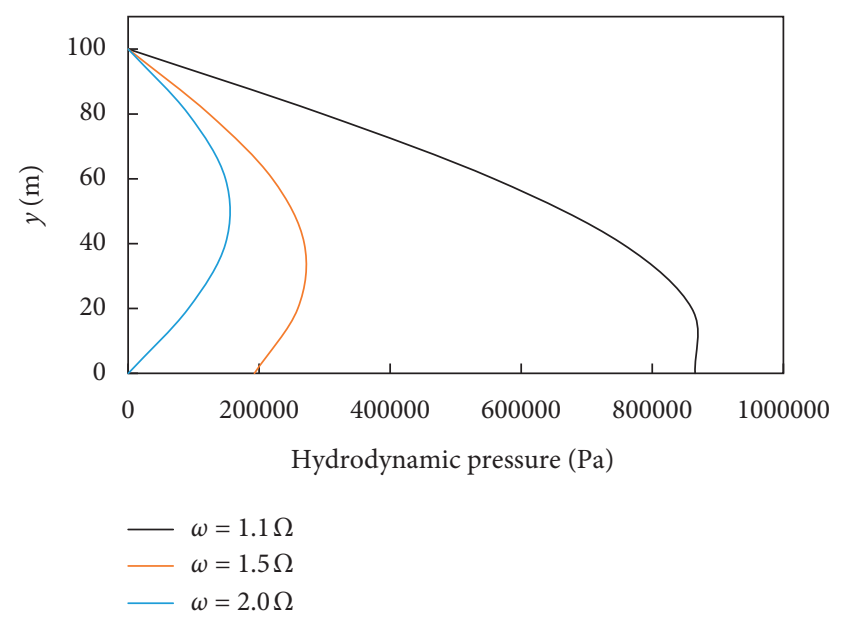

FIGURE 5: Pressure distribution along the dam height for frequencies more than the natural frequency of the reservoir in flexible foundation case.

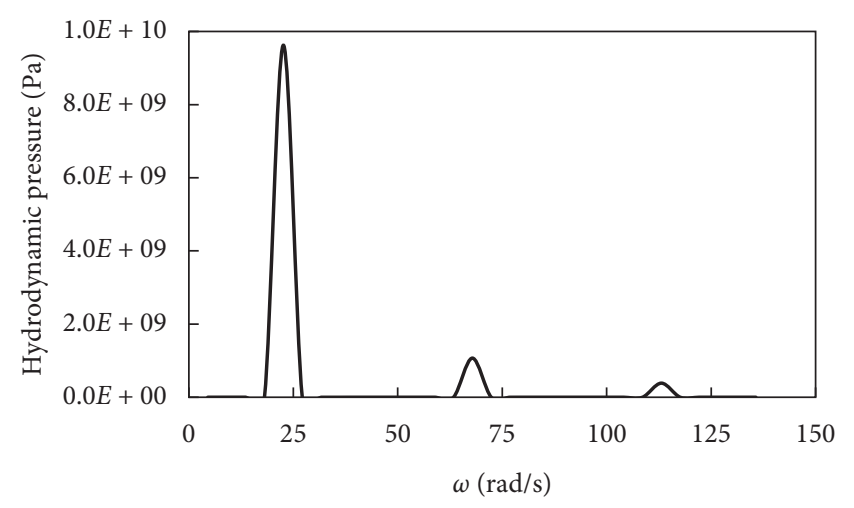

FIGURE 6: The effect of excitation frequency on the pressure distribution at the bottom of the reservoir for the rigid foundation.

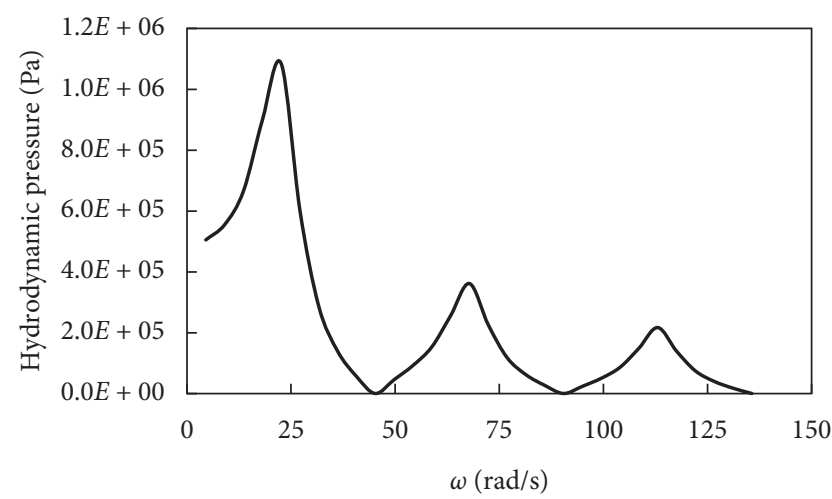

FIgURE 7: The effect of excitation frequency on the pressure distribution at the bottom of the reservoir for the flexible foundation.

In this study, the hydrodynamic response of a concrete gravity dam and reservoir under vertical excitation was investigated using an analytical solution. The results showed that the distribution of hydrodynamic pressure due to vertical vibration is not dependent on the longitudinal direction, and the propagation of hydrodynamic wave occurs only along the vertical direction. For an incompressible fluid, the pressure distribution along the dam is linear.

Based on the results, it can be found that for the excitation frequencies more than the natural frequency of the reservoir, unlike the excitation frequencies less than the natural frequency of the reservoir, the maximum hydrodynamic pressure does not occur in the bottom of the reservoir. The case in which $\omega=n \Omega$, the hydrodynamic pressure in the bottom of the reservoir is zero, which is due to absorption of excitation by the natural vibration of the reservoir. Also, the study of pressure distribution curves indicates that the compressibility effects for the excitation frequency are more than the case in which the frequency is equal to half the natural frequency of the reservoir. The results also show the sensitivity of the response to the loading frequency. According to the sensitivity analysis of the response to the excitation frequency, it can be seen that the maximum responses are related to the conditions where the excitation frequency is close to the natural frequencies of the reservoir.

\section{Data Availability}

The data used to support the findings of this study are included within the article.

\section{Conflicts of Interest}

The authors declare that they have no conflicts of interest.

\section{References}

[1] H. M. Westergaard, "Water pressures on dams during earthquakes," Transactions of the American Society of Civil Engineers, vol. 98, no. 2, pp. 418-433, 1933.

[2] A. K. Chopra, "Hydrodynamic pressures on dams during earthquakes," Journal of the Engineering Mechanics Division, vol. 93, no. 6, pp. 205-223, 1967.

[3] A. K. Chopra, "Earthquake behavior of reservoir-dam systems," Journal of the Engineering Mechanics Division, vol. 94, no. 6, pp. 1475-1500, 1968.

[4] A. K. Chopra, "Earthquake response of concrete gravity dams," Journal of the Engineering Mechanics Division, vol. 96, no. 4, pp. 443-454, 1970.

[5] B. Nath, "Structural and hydrodynamic coupling for a gravity dam during vertical earthquake motions," Dynamic Wave in Civil Engineering, pp. 423-440, Wiley-Interscience, Hoboken, NJ, USA, 1971.

[6] P. Chakrabarti and A. K. Chopra, "Hydrodynamic pressures and response of gravity dams to vertical earthquake component," Earthquake Engineering and Structural Dynamics, vol. 1, pp. 325-335, 1973.

[7] J. L. Humar and A. M. Jablonski, "Boundary element reservoir model for seismic analysis of gravity dams," Earthquake Engineering \& Structural Dynamics, vol. 16, no. 8, pp. 11291156, 1988.

[8] M. Ghaemian, A. H. Vafai, and Z. Karimi, "Nonlinear seismic response of concrete gravity dams due to foundation fault movement," Scientia Iranica, vol. 21, no. 5, pp. 1539-1548, 2014. 
[9] S. H. Ziaolhagh, M. Goudarzi, and A. A. Sani, "Free vibration analysis of gravity dam-reservoir system utilizing 21 node-33 Gauss point triangular elements," Coupled Systems Mechanics, vol. 5, no. 1, pp. 59-86, 2016.

[10] P. M. V. Ribeiro and L. J. Pedroso, "Dynamic Response of dam-reservoir systems: review and a semi-analytical proposal," Latin American Journal of Solids and Structures, vol. 14, no. 4, pp. 594-612, 2017.

[11] Z. Shah, L. B. McCash, A. Dawar, and E. Bonyah, "Entropy optimization in Darcy-Forchheimer MHD flow of water based copper and silver nanofluids with Joule heating and viscous dissipation effects," AIP Advances, vol. 10, no. 6, p. 065137, 2020.

[12] Z. Shah, M. R. Hajizadeh, I. Ikramullah, N. A. Alreshidi, W. Deebani, and M. Shutaywi, "Entropy optimization and heat transfer modeling for Lorentz forces effect on solidification of NEPCM," International Communications in Heat and Mass Transfer, vol. 117, p. 104715, 2020.

[13] Z. Shah, M. Sheikholeslami, and S. Ikramullah, P. Kumam, Influence of nanoparticles inclusion into water on convective magneto hydrodynamic flow with heat transfer and entropy generation through permeable domain," Case Studies in Thermal Engineering, vol. 21, p. 100732, 2020.

[14] A. Dawar, Z. Shah, P. Kumam et al., "Chemically reactive MHD micropolar nanofluid flow with velocity slips and variable heat source/sink," Science Reports, vol. 10, p. 20926, 2020.

[15] A. S. Khan, N. Nie, Z. Shah et al., "Influence of interfacial electrokinetic on MHD radiative nanofluid flow in a permeable microchannel with Brownian motion and thermophoresis effects," Open Physics, vol. 18, no. 1, 2020.

[16] S. Nasir, Z. Shah, W. Khan, H. Alrabaiah, S. Islam, and S. N. Khan, "MHD stagnation point flow of hybrid nanofluid over a permeable cylinder with homogeneous and heterogenous reaction," Physica Scripta, vol. 96, no. 3, p. 96, Article ID 03520, 2021.

[17] G. Fenves and A. K. Chopra, "Effects of reservoir bottom absorption and dam-water-foundation rock interaction on frequency response functions for concrete gravity dams," Earthquake Engineering and Structural Dynamics, vol. 13, no. 1, pp. 13-31, 1985.

[18] R. Malek-Madani, Advanced Engineering Mathematics with Mathematica and MATLAB, Vol. 1, Pearson Education, Reading, MA, USA, 1997.

[19] A. K. Chopra and P. Chakrabarti, "Earthquake analysis of concrete gravity dams including dam-water-foundation rock interaction," Earthquake Engineering and Structural Dynamics, vol. 9, pp. 363-383, 1982.

[20] M. Ghaemian and A. Ghobarah, "Staggered solution schemes for dam-reservoir interaction," Journal of Fluid and Structures, vol. 12, pp. 933-948, 1998. 